\title{
Expression of PCNA in oral gingival epithelium of aggressive and, chronic periodontitis-A pilot study
}

\author{
Penapy Jyostna ${ }^{1}$, Manchala Sesha Reddy ${ }^{2 *}$, Manyam Ravikanth ${ }^{1}$, Mandalapu Narendra ${ }^{1}$, \\ Chini Dorasani Dwarakanath ${ }^{1}$ \\ ${ }^{1}$ Vishnu Dental College, Bhimivaram, India \\ ${ }^{2}$ Gulf Medical University, Ajman, UAE \\ Email: bujji0122@gmail.com, ${ }^{*}$ seshaperio5@gmail.com, drravikanth@gmail.com, plaque@rediffmail.com, drdwarakcd@yahoo.co.in
}

Received 30 January 2013; revised 2 March 2013; accepted 15 March 2013

\begin{abstract}
Periodontal disease leads to loss of tissue homeostasis of tooth supporting structures. The mechanism of fibrous tissue degradation by various enzymes in chronic periodontitis and aggressive periodontitis was well documented but there is paucity of information regarding epithelial response. The aim of the present study was to evaluation of epithelial cell proliferation in Chronic Periodontitis, Aggressive Periodontitis and normal patients using Proliferating Cell Nuclear Antigen (PCNA). The study consists of 30 individuals who attended the Periodontia clinic of Vishnu Dental College. They were divided equally into 3 groups: chronic periodontitis (10) aggressive periodontitis (10) and normal individuals requiring gingival excision for crown lengthening (10). Excised gingival tissues were routinely processed and subjected to Immunohistochemical evaluation using Proliferating Cell Nuclear Antigen (PCNA). The mean proliferation index was almost equal in Aggressive periodontitis patients (36.55) and normal individuals (37.02) but it was high in Aggressive Periodontitis patients when compared to chronic periodontitis group (18.86). Observations of this study indicate the possibility of subclinical inflammation in clinically normal gingiva and difference in the types of microorganism associated with chronic periodontitis and aggressive periodontitis.
\end{abstract}

Keywords: Immunohistochemistry; Normal Gingiva; Aggressive Periodontitis; Chronic Periodontitis; Mitotic Index; Proliferating Cell Nuclear Antigen

\section{INTRODUCTION}

Periodontal disease is caused by constant exposure of periodontal tissue to bacteria finally leading to destruction of the tooth supporting tissues and loss of teeth. It is

"Corresponding author. a chronic condition characterized by periods of active tissue destruction at specific sites followed by healing $[1,2]$. Periodontal epithelium serves to cover and protect the underlying tissues. In this respect, the local immune response and presence of a high cell turnover in the gingival epithelium are important for establishing and maintaining a host-microorganism equilibrium [3].

Proliferating Cell Nuclear Antigen (PCNA) is found in cells of proliferation compartment of normal tissue. PCNA is a 36-kDa auxiliary protein, plays an essential role in nucleic acid metabolism as a component of replication and repair machinery. This protein is essential for DNA replication and elevated in nucleus during late G1 phase, immediately before the onset of DNA synthesis: expression becomes maximal during the $\mathrm{S}$ phase before declining during $\mathrm{G} 2$ and $\mathrm{M}$ phases [4,5]. Although PCNA positive cells were observed in both basal and suprabasal layers, it is widely accepted that proliferation of gingival epithelial cells take place by mitosis in basal layer and less frequently in suprabasal layers [6].

Chronic periodontitis and aggressive periodontitis share the clinical features of bone loss and clinical attachment loss in response to colonization of the tooth surface by a bacterial film. However the difference lies in the pattern and rate of disease progression. Though connective tissue changes of chronic periodontitis and aggressive periodontitis are well documented, the status of epithelial component is not well described, especially from a histological perspective [7].

The present study was undertaken to evaluate the epithelial cell proliferation in Chronic Periodontitis, Aggressive Periodontitis patients and normal patients using PCNA.

\section{MATERIALS AND METHODS}

\subsection{Patient Selection}

A total of 30 subjects (15 males and 15 females) selected from the patient pool attending the outpatient clinic, De- 
partment of Periodontics, Vishnu Dental College and Hospital, Bhimavaram. The institutional review board of Vishnu Dental College approved the study protocol and informed consent was obtained from each subject. Subjects were divided into three groups. Group A comprised of 10 subjects with clinically normal and healthy gingiva, with no clinical signs of inflammation, who were subjected for crown lengthening procedure. Group B comprised of 10 subjects diagnosed clinically and radiographically as Aggressive Periodontitis, Group C comprised of 10 subjects diagnosed clinically and radiographically as Chronic Periodontitis, both groups were subjected for flap surgery.

\subsection{Tissue Collection}

All gingival biopsies obtained from the subjects were fixed in $10 \%$ neutral buffered formalin and routinely processed. $4 \mu \mathrm{m}$ thick sections were made from the paraffin blocks and mounted on 3 aminopropyltriethoxysilane coated slides.

\section{IMMUNOHISTOCHEMISTRY}

The tissues were deparaffinized by two changes in $x y-$ lene (10 minutes) each, placed in descending grades of alcohol and then rehydrated with water. They were then transferred to citrate buffer and antigen retrieval was done using microwave method, 3 cycles 5 min each at power $450 \mathrm{~W}$. Then the slides were dipped in two changes of phosphate buffered saline (PBS) for 5 minutes and then wiped carefully with gauge to remove excess PBS. The slides were treated with 3\% hydrogen peroxide for 10 minutes, and put in two changes of PBS and then treated with power block for 20 minutes.

The primary antibody PC10 (BioGenex) were added to tissue samples and was incubated at room temperature for 1 hour. The sections were taken out and washed in two changes of cold PBS (10 minutes each), a drop of super enhancer was added and the slides were incubated for 30 minutes. The slides were then washed in two changes of PBS 10 minutes each. Secondary antibody was added and incubated for 30 minutes. Sections were washed and carefully wiped to remove excess PBS and a drop of freshly prepared DAB (3' Damino Benzadine Tetra Hydrochloride - a substrate chromogen) was added onto sections. Slides were then washed in running distilled water to remove excess DAB and counter stained with Harri's hematoxylin, later washed with acid alcohol and xylene. Tissue sections were mounted with Disterene dibutylphthalate (DPX) for microscopic examination at magnification $40 \times$.

\section{EVALUATION}

Images of five random fields were captured using BX 51 microscope with 0.5 adapter, $40 \times$ lens and DP 71 camera. PCNA expression was evaluated by counting the number of cells that were stained in brown color and given the color coding of red circle for proliferating cells and green color dot for non proliferating cells. Proliferation index was calculated by dividing the number of positively stained cells with the total number of cells in the magnified section. Data entry and descriptive analysis was performed using the SPSS version 10.0 statistical software program. Paired T-Test was done for statistical analysis.

\section{RESULTS}

All tissues examined showed the presence of oral gingival epithelium (E) and subjacent connective tissue (CT) Figures 1 and 2. Cell counting has been carried out in five sections per tissue slide and the mean was calculated. The mean mitotic index in the epithelium of normal gingiva with Aggressive periodontitis and chronic periodontitis patients was compared by using paired t-test. (Table 1). Figures 1 and 2 illustrates the immunohistochemical staining of gingival tissue samples of normal patients (Group A), Aggressive Periodontitis patients (Group B), Chronic Periodontitis patients (Group C) before cell counting (A, B, C) and after cell counting (a, b, c) respectively. Table 1 and Graph 1 show the comparison of mitotic activity within groups, with a statistical significant difference between groups Normal and Chronic Periodontitis groups with a p value of $<0.05$ and between Aggressive Periodontitis and Chronic Periodontitis groups with a p value $<0.005$.

\section{DISCUSSION}

Periodontitis is a common oral disease initiated and per-

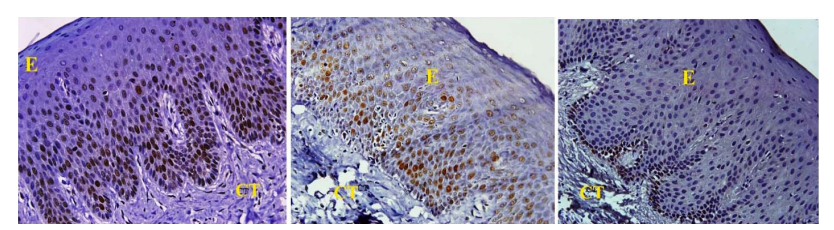

(a)

(b)

(c)

Figure 1. PCNA staining in epithelium and connective tissue before cell counting in (a) Normal; (b) Aggressive periodontitis; (c) Chronic periodontitis gingiva.

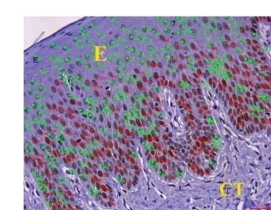

(a)

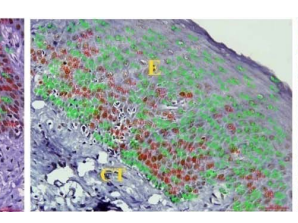

(b)

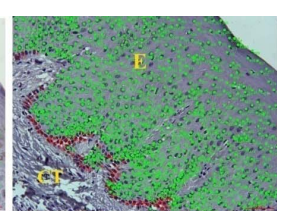

(c)
Figure 2. PCNA staining in epithelium and connective tissue after cell counting in (a) Normal; (b) Aggressive periodontitis; (c) Chronic periodontitis gingiva. 
Table 1. Compression of mitotic index in normal, aggressive and chronic periodontitis groups using paired t-test.

\begin{tabular}{lccc}
\hline Mitotic index & Sample size & Mean \pm SD & p value \\
\hline Normal & 10 & $37.02 \pm 9.49$ & 0.929 \\
Aggressive periodontitis & 10 & $36.55 \pm 13.53$ & Not significant \\
Normal & 10 & $37.02 \pm 9.49$ & \\
Chronic periodontitis & 10 & $18.86 \pm 10.07$ & $0.005^{*}$ \\
Aggressive periodontitis & 10 & $36.55 \pm 13.53$ & $0.016^{\#}$ \\
Chronic periodontitis & 10 & $18.86 \pm 10.07$ & \\
\hline
\end{tabular}

${ }^{*}<0.05$ significant; ${ }^{*}<0.005$ significant.

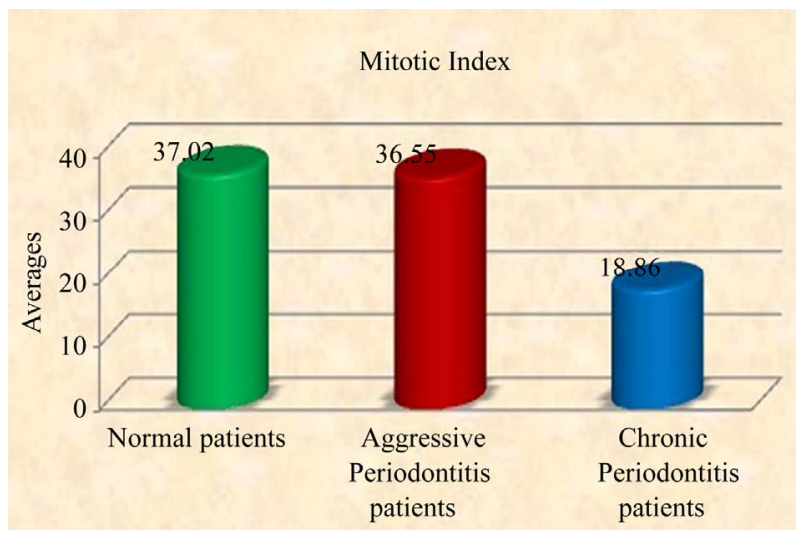

Graph 1. Graphic representation of mitotic index in normal, aggressive and chronic periodontitis group.

petuated by a group of predominantly gram-negative anaerobic bacteria, such as Porphyromonas gingivalis, Bacteroides forsythus and Actinobacillus actinomycetemcomitans [8]. The ability of gingival sulcular and junctional epithelia to control bacterial challenge and prevent tissue destruction is crucial for maintenance of periodontal health. At the same time gingival epithelium provides the protective integument of the periodontium. The histological appearance of human gingival epithelium has been studied in great detail and described by numerous authors [9-13]. This study report characterizes the epithelial cell behavior collected from clinically healthy and inflamed human gingival tissues.

Authors identified an auto-antigen in patients with systemic lupus erythematosis, which was named as PCNA because the protein was observed in the nucleus of dividing cells [14]. The advantage in using PCNA is that it stains the proliferating cell even though they are in various stages of cell cycle and in various layers of epithelium [6]. Various researchers used PCNA for evaluation of epithelial cell proliferation and they considered PCNA as a reliable indicator of proliferation $[15,16]$. This study was the first to assess epithelial cell proliferation in gingival biopsies of Normal, Aggressive and Chronic Periodontitis patients using PCNA. P. gingivalis and A. ac- tinomycetemcomitans are the two most studied periodontal pathogens. Slots \& Genco [17] reported that an array of bacterial virulence factors enable these two bacteria to colonize periodontal sites, evade host defenses and break down periodontal tissue. Holt et al. [18] suggested that $P$. gingivalis excretes an abundance of potent proteases, including a collagenase and a trypsin-like enzyme. Fives-Taylor et al. [19] have been proposed that $A$. actinomycetemcomitans elaborates a variety of toxic products such as a potent endotoxin, a leukotoxin, a cytotoxin and immunosuppressive factors. Furthermore, $P$. gingivalis and $A$. actinomycetemcomitans seem to constitute exogenous microorganisms that are transmitted via saliva among individuals and may cause disease in susceptible hosts after exceeding certain threshold levels of the organisms.

The present study indicates a statistically significant difference between Normal and CP groups with p value $<0.05$, suggesting the low mitotic index in chronic periodontitis group which could be due to degree of inflammation that is slowly progressive with periods of quiescence and exacerbation. Noiri et al. [20] observed bacteria within epithelial cells in tissue from patients with advanced adult periodontitis. Cutler et al also presented evidence that $P$. gingivalis was taken up by dendritic cells in the gingiva of an adult periodontitis patient [21]. Thus, it can be theorized that the invasion mechanism of $P$. gingivalis is dependent on the surface recaptors and intracellular signal transduction pathways available in specific host cells.

The modulation of epithelial cell signal transduction pathways by $P$. gingivalis can have an impact on gene expression, through, for example, affects on the transcriptional activator NF-kB [22]. The gene encoding the neutrophil chemokine interleukin-8 is transcriptionally down-regulated by $P$. gingivalis even in the presence of otherwise stimulatory organisms such as $F$. nucleatum [23-26]. Inhibition of interleukin-8 accumulation by $P$. gingivalis at sites of bacterial invasion could have a debilitating effect on innate host defense at the bacteriaepithelia interface. The host would no longer be able to detect the presence of bacteria and direct neutrophils for their removal. In the normal healthy gingiva, the tissue homeostasis is maintained and the mitotic index equalizes with apoptotic index.

Given that gingival epithelial cells turn over about every 5 - 7 days in vivo, a significant period of their existence could be in association with internalized bacteria. Nevertheless, the ultimate death of the host cell is an event that looms large in the future of intracellular bacteria. A. actinomycetemcomitans and $P$. gingivalis have developed different strategies to deal with this problem. A. actinomycetemcomitans can jump from one cell to another [27] thereby minimizing the effect of epithelial 
cell death.

$P$. gingivalis is able to inhibit apoptotic death in gingival epithelial cells. This is accomplished through induction of the anti-apoptotic protein Bcl-2 and suppression of the pro-apoptotic protein Bax [28]. Thus, having located in a nurturing environment, $P$. gingivalis prolongs the life of its host. $P$. gingivalis is also able to antagonize the effects of apoptosis inducing agents such as camptothecin. The ability to interfere with programmed cell death could have significant repercussions for the normal development, integrity and homeostasis of periodontal tissues and their by having an effect on the reduction of mitotic index.

The study also revealed a statistically significant difference between groups AP and CP group with p value of 0.016 , which can be explained on the basis of the rate of disease progression which is higher in aggressive periodontitis than chronic form. The main organism behind the disease progression is A. actinomycetocomitans. In the case of A. actinomycetemcomitans, invaded epithelial cells are induced to secrete interleukin-8 and to upregulate intercellular adhesion molecule-1 [20,29]. $A$. actinomycetemcomitans may be able to avoid the encounter with immune effectors by remaining intracellular and spreading from cell to cell through the interconnecting intercellular protrusions. The immune response may then burn out leaving a situation, as observed clinically, whereby localized juvenile periodontitis generally does not involve significant inflammation. Its toxin is a repeats-in-toxin responsible for rapid tissue destruction. The bacteria may escape the anti-bacterial actions of the immune system by surviving within epithelial cells and other periodontal mammalian cells. To this bacterial activity, host responds for bacterial elimination by shedding off the epithelial cells containing bacteria into oral cavity, and formation of new cells through proliferation [30]. This in turn may be a cause of high mitotic index in AP group when compared to CP group.

All these factors have influence on the proliferating activity for the maintenance of balance between the invader and defender. Pro-inflammatory cytokine expression and lipopolysaccharides from Gram-negative microorganisms are associated with disease function, directly or indirectly and modulate the cellular behavior. Pro-inflammatory cytokines (interleukin-1a, interleukin$1 \mathrm{~b}$, interleukin- 6 and tumor necrosis factor- $\alpha$ and purified lipopolysaccharide from gram negative bacteria, all significantly induced gingival fibroblast KGF-1 gene and protein expression [31]. This peculiar feature can be implicated to our results as high mitotic index values (36.55 \pm 13.53 ) was seen in AP group which indicate that the aggressive periodontitis patents show a rapid rate of periodontal destruction, with the presence of tissue invading bacteria.
Our data suggested little or no statistical significant difference between Normal and AP groups with value of 0.929 (Table 1). A few of the samples from Normal group also showed values nearing those of AP group (Graph 1) which can be attributed to the subclinical levels of inflammation in clinically healthy tissues. Clinically healthy gingiva may show a degree of subclinical inflammation. Since we cannot obtain pristine gingiva from normal human beings, this would be a limitation to study which has to be further considered.

A contradictory statement was reported by an in vitro study conducted by Carro O.M. et al. [32]. They obtained an inverse relation of mitotic index with the degree of inflammation. Since it is an in vitro study all the cell cultures, individual cells in each culture may respond to inflammation by increased proliferation but quickly followed by cell death. Once a putative threshold level is exceeded the cells may lose their adaptive capacity and consequently enter into a pathologic condition that may be linearly associated with the degree of inflammation.

\section{CONCLUSION}

The study revealed a high mitotic index in aggressive periodontitis compared to chronic periodontitis gingival samples however; the proliferating rate of aggressive periodontitis and the normal gingiva are similar suggesting a possible subclinical inflammation of the normal gingival sample. Though the degree of inflammation and presence of tissue penetrating bacteria has a key role behind the periodontal disease progression, a further evaluation, by combining various aspects regulating the cell turnover like the mitotic activity, human $\beta$-defensins, apoptotic activity, the genes responsible for these processes and various growth factors expression would give better results.

\section{REFERENCES}

[1] Dale, B.A. (2002) Periodontal epithelium: A newly recognized role in health and disease. Periodontology, 30, 70-78. doi:10.1034/j.1600-0757.2002.03007.x

[2] Darveau, R.P., Tanner, A. and Page, R.C. (1997) The microbial challenge in periodontitis. Periodontology, 14, 12-32. doi:10.1111/j.1600-0757.1997.tb00190.x

[3] Genco, R. (1992) Host responses in periodontal diseases: Current concepts. Journal of Periodontology, 63, 338-355. doi:10.1902/jop.1992.63.4s.338

[4] Jain, S., Filipe, M.I., Hall, P.A., Waseem, N., Lane, D.P. and Levison, D.A. (1991) Prognostic value of proliferating cell nuclear antigen in gastric carcinoma. Journal of Clinical Pathology, 44, 655-659. doi:10.1136/jcp.44.8.655

[5] Gultekin, S.E., Senguven, B. and Karaduman, B. (2008) Effect of smoking on epithelial proliferation in healthy and periodontally diseased marginal gingival epithelium. 
Journal of Periodontology, 79, 1444-1450. doi:10.1902/jop.2008.070645

[6] Kelman, Z. (1997) PCNA: Structure, function and interactions. Oncogene, 14, 629-640.

doi:10.1038/sj.onc.1200886

[7] Seymour, G.J., Powell, R.N. and Davies, W.I.R. (1979) Conversion of a stable $\mathrm{T}$-cell lesion to a progressive B-cell lesion in the pathogenesis of chronic inflammatory periodontal disease: and hypothesis. Journal of Clinical Periodontology, 6, 267-277. doi:10.1111/j.1600-051X.1979.tb01930.x

[8] Consensus Report (1996) Periodontal diseases: Pathogenesis and microbial factors. Annals of Periodontology, 1, 926-932.

[9] Muller-Glauser, W. and Schroeder, H. (1983) Exfoliative cytology and ultrastructure of superficial epithelial cells from the normal human oral stratified epithelia. Journal de Biologie Buccale, 11, 317-326.

[10] Schroeder, H. and Theilade, J. (1996) Electron microscopy of normal human gingival epithelium. Journal of Periodontal Research, 1, 95-119. doi:10.1111/j.1600-0765.1966.tb01850.x

[11] Schroeder, H. (1981) Differentiation of human oral stratified epithelia. Karger, Basel, 35-119.

[12] Schroeder, H.E. and Listagarten, M. (1997) The gingival tissues: The architecture of periodontal protection. $\mathrm{Pe}$ riodontology, 13, 91-120. doi:10.1111/j.1600-0757.1997.tb00097.x

[13] Hassel, M.T. (1993) Tissues and cells of periodontium. Periodontology, 3, 9-38. doi:10.1111/j.1600-0757.1993.tb00230.x

[14] Miyachi, K. and Fritzler, M.J. (1978) Autoantibody to a nuclear antigen in proliferating cells. Journal of Immunology, 121, 2228-2234.

[15] Acikgoz, G., Devrim, I. and Ozdamar, S. (2004) Comparison of keratinocyte proliferation in diabetic and non diabetic inflamed gingiva. Journal of Periodontology, 75, 989-994. doi:10.1902/jop.2004.75.7.989

[16] Nurmenniemi, P.K., Pernu, H.E. and Knuuttila, M.L.E. (2001) Mitotic activity of keratinocytes in nifidipine and immunosuppressive medication induced gingival overgrowth. Journal of Periodontology, 72, 167-173. doi:10.1902/jop.2001.72.2.167

[17] Slots, J. and Genco, R.J. (1984) Black-pigmented Bacteroides species, Capnocytophaga species, and Actinobacillus actinomycetemcomitans in human periodontal disease: Virulence factors in colonization, survival, and tissue destruction. Journal of Dental Research, 63, 412-421. doi:10.1177/00220345840630031101

[18] Holt, S., Kesavalu, L., Walker, S. and Genco Attardo, C. (1999) Virulence factors of Porphyromonas gingivalis. Periodontology, 20, 168-238. doi:10.1111/j.1600-0757.1999.tb00162.x

[19] Fives-Taylor, P., Hutschins Meyer, D., Mintz, K.P. and Brissette, C. (1999) Virulence factors of Actinobacillus actinomycetemcomitans. Periodontology, 20, 136-167. doi:10.1111/j.1600-0757.1999.tb00161.X

[20] Noiri, Y., Ozaki, K., Nakae, H., Matsuo, T. and Ebisu, S.
(1997) An immunohistochemical study on the localization of Porphyromonas gingivalis, Campylobacter rectus and Actinomyces viscosus in human periodontal pockets. Journal of Periodontal Research, 32, 598-607. doi:10.1111/j.1600-0765.1997.tb00937.x

[21] Cutler, C.W., Jotwani, R., Palucka, K.A., Davoust, J., Bell, D. and Banchereau, J. (1999) Evidence and a novel hypothesis for the role of dendritic cells and Porphyromonas gingivalis in adult periodontitis. Journal of Periodontal Research, 34, 406-412. doi:10.1111/j.1600-0765.1999.tb02274.X

[22] Watanabe, K., Yilmaz, O., Nakhjiri, S.F., Belton, C.M. and Lamont, R.J. (2001) Association of mitogen-activated protein kinase pathways with gingival epithelial cell responses to Porphyromonas gingivalis infection. Infection and Immunity, 69, 6731-6737. doi:10.1128/IAI.69.11.6731-6737.2001

[23] Darveau, R.P., Belton, C.M., Reife, R.A. and Lamont, R.J. (1998) Local chemokine paralysis, a novel pathogenic mechanism for Porphyromonas gingivalis. Infection and Immunity, 66, 1660-1665.

[24] Huang, G.T., Haake, S.K., Kim, J.W. and Park, N. (1998) Differential expression of interleukin-8 and intercellular adhesion molecule-1 by human gingival epithelial cells in response to Actinobacillus actinomycetemcomitans or Porphyromonas gingivalis infection. Oral Microbiology and Immunology, 13, 301-309. doi:10.1111/j.1399-302X.1998.tb00711.x

[25] Huang, G.T., Kim, D., Lee, J.K., Kuramitsu, H.K. and Kinder Haake, S. (2001) Interleukin-8 and intercellular adhesion molecule 1 regulation in oral epithelial cells by selected periodontal bacteria. Multiple effects of Porphyromonas gingivalis via antagonistic mechanisms. Infection and Immunity, 69, 1364-1372. doi:10.1128/IAI.69.3.1364-1372.2001

[26] Madianos, P.N., Papapanou, P.N. and Sandros, J. (1997) Porphyromonas gingivalis infection of oral epithelium inhibits neutrophil transepithelial migration. Infection and Immunity, 65, 3983-3990.

[27] Meyer, D.H., Rose, J.E., Lippmann, J.E. and Fives-Taylor, P.M. (1999) Microtubules are associated with intracellular movement and spread of the periodontopathogen Actinobacillus actinomycetemcomitans. Infection and Immunity, 67, 6518-6525.

[28] Nakhjiri, S.F., Park, Y., Yilmaz, O., Chung, W.O., Watanabe, K., El-Sabaeny, A., Park, K and Lamont, R.J. (2001) Inhibition of epithelial cell apoptosis by $P$. gingivalis. FEMS Microbiology Letters, 200, 145-149. doi:10.1111/j.1574-6968.2001.tb10706.x

[29] Huang, G.T., Haake, S.K. and Park, N. (1998) Gingival epithelial cells increase interleukin-8 secretion in response to Actinobacillus actinomycetemcomitans challenge. Journal of Periodontology, 69, 1105-1110. doi:10.1902/jop.1998.69.10.1105

[30] Slots, J. (1999) Actinobacillus actinomycetemcomitans and Porphyromonas gingivalis in periodontal disease: Introduction, Periodontology, 20, 7-13. doi:10.1111/j.1600-0757.1999.tb00155.x

[31] Li, M., Frith, J.D. and Putnins, E.E. (2005) Keratinocyte 
growth factor 1 expression in healthy and diseased human periodontal tissues. Journal of Periodontal Research, 40, 118-128. doi:10.1111/j.1600-0765.2004.00780.x

[32] Carro, O.M., Evans, S.A.S. and Leone, C.W. (1997) Ef- fect of inflammation on the proliferation of human gingival epithelial cells in vitro. Journal of Periodontology, 68, 1. doi:10.1902/jop.1997.68.11.1070 